\title{
Parameterisation effect on the behaviour of a head-dependent hydro chain using a nonlinear model
}

\author{
J.P.S. Catalão ${ }^{\mathrm{a}, *}$, S.J.P.S. Mariano ${ }^{\text {a }}$, V.M.F. Mendes ${ }^{\mathrm{b}}$, L.A.F.M. Ferreira ${ }^{\mathrm{c}}$ \\ ${ }^{a}$ Department of Electromechanical Engineering, University of Beira Interior, R. Fonte do Lameiro, 6201-001 Covilha, Portugal \\ ${ }^{\mathrm{b}}$ Department of Electrical Engineering and Automation, Instituto Superior de Engenharia de Lisboa, \\ R. Conselheiro Emídio Navarro, 1950-062 Lisbon, Portugal \\ ${ }^{\mathrm{c}}$ Department of Electrical Engineering and Computers, Instituto Superior Técnico, Technical University of Lisbon, \\ Av. Rovisco Pais, 1049-001 Lisbon, Portugal
}

Received 5 March 2005; received in revised form 13 July 2005; accepted 1 September 2005

Available online 3 October 2005

\begin{abstract}
This paper is on the problem of short-term hydro scheduling (STHS), particularly concerning a head-dependent hydro chain. We use a method based on nonlinear programming (NLP), namely quadratic programming, to consider hydroelectric power generation a function of water discharge and of the head. The method has been applied successfully to solve a test case based on a realistic cascaded hydro system with a negligible computational time requirement and is also applied to show that the role played by reservoirs in the hydro chain do not depend only on their relative position. As a new contribution to earlier studies, which presented reservoir operation rules mainly for medium and long-term planning procedures, we show that the physical data defining hydro chain parameters used in the nonlinear model have an effect on the STHS, implying different optimal storage trajectories for the reservoirs accordingly not only with their position in the hydro chain but also with the new parameterisation defining the data for the hydro system. Moreover, considering head dependency in the hydroelectric power generation, usually neglected for hydro plants with a large storage capacity, provides a better short-term management of the conversion of the potential energy available in the reservoirs into electric energy, which represents a major advantage for the hydroelectric utilities in a competitive electricity market.
\end{abstract}

(C) 2005 Elsevier B.V. All rights reserved.

Keywords: Short-term hydro scheduling (STHS); Nonlinear programming (NLP); Cascaded reservoirs; Variable head

\section{Introduction}

In this paper, the short-term hydro scheduling (STHS) problem of a head-dependent hydro chain is considered. Hydro plants with a small storage capacity are known as run-of-the-river. Typically, run-of-the-river hydro plants are considered to operate under stationary conditions with constant head and at the maximum water level in the reservoirs, corresponding by design to the optimum efficiency operating point. However, it is often desirable to change this policy, thus incurring into head changes. The operating efficiency is sensitive to the head-head change effect.

Significant loss of efficiency can occur in operating hydro plants away from their most efficient operating point. Thus, hydroelectric power generation has to be considered as a

\footnotetext{
* Corresponding author.

E-mail addresses: catalao@ubi.pt (J.P.S.Catalão),sm@ubi.pt (S.J.P.S. Mariano), vfmendes@isel.pt (V.M.F. Mendes), lmf@ist.utl.pt (L.A.F.M. Ferreira).
}

function of water discharge and also of the head in order to avoid this loss of efficiency, that is, in order to take in account the head change effect. In a run-of-the-river cascaded hydraulic configuration an upstream reservoir highly influences the operation of the next downstream reservoir. The latter reservoir also influences the upstream plant by its effect on the tail water elevation and effective head [1]. Actually, the cascaded hydraulic configuration coupled with the nonlinear head change effect, augments the problem dimension and the complexity, but they should be considered because they are important for the most advantage management of the conversion of the potential energy available in the reservoirs into electric energy.

In a competitive electricity market, the most advantageous management of the conversion of the potential energy available in the reservoirs into electric energy is not only a major advantage for the hydroelectric utilities, but also is essential for the welfare, development and the economic progress of our society. This is because hydroelectric energy can be regarded as a renewable 
source of energy fuelled by the sun. Furthermore, contributes to reduce the use of fossil fuels energy, which is costly, and avoids unnecessary environmental damage due to pollutant emissions coming from thermal plants [2].

Therefore, we need to consider the importance of improving STHS models as a powerful aid for a better management. In the STHS optimisation problem a time horizon of 1-7 days is considered, usually discretized in hourly periods. Hence, the STHS problem is treated as a deterministic one. Where the problem includes stochastic quantities such as inflows to reservoirs or energy prices, the corresponding forecasts are used. STHS is guided by specified hourly weighting factors, quantifying the energy price at each hour [3]. The goal is to maximize the value of total hydroelectric power generation throughout the time horizon, satisfying all physical and operational constraints, and consequently to maximize the profit of the hydroelectric utility from selling energy into the electric market [4].

Many methods have been used to solve the STHS problem. Dynamic programming (DP) is among the earliest methods applied to the STHS problem [5-8]. Although DP can handle the non-convexities and the nonlinear characteristics present in the hydro model, it suffers from the well-known curse of dimension, more difficult to avoid in short-term than in long-term optimisation without losing the accuracy needed in the model [9].

Artificial intelligence techniques have also been successfully applied to the STHS problem, namely, neural networks $[10,11]$ and genetic algorithms [12,13]; although there are some problems concerning the computational effort necessary to solve the problem.

A natural approach to STHS is to model the system as a network flow model [14-16], because of the underlying network structure subjacent in hydro chains. This network flow model is often programmed as a linear or piecewise linear one. Linear programming (LP) is a well-known optimisation method and standard software is available [17-19]. Also, mixed-integer linear programming (MILP) is becoming frequently used for hydro scheduling [20-24], where binary variables allow modelling of start-up costs to avoid unnecessary start-ups, and of discrete hydro unit-commitment constraints. However, LP typically considers that power generation is linearly dependent on water discharge, thus ignoring the head change effect, leading to a solution schedule with less power generation. Also, the discretization of the nonlinear dependence between power generation, water discharge and head, used in MILP to model head variations, augment the computational burden required to solve this problem [24].

A nonlinear model has advantages compared with a linear one. A nonlinear model expresses hydroelectric power generation characteristics more accurately and the head change effect can be taken into account $[9,25]$.

In this paper, we use a nonlinear programming (NLP) method to solve the STHS problem in a market environment. A comparison between this NLP method with DP for a case study consisting of a single hydro plant, and with LP that ignores the head change effect, was shown in [4], illustrating the success and the benefits of the method, improving the results of STHS particularly in reservoirs where the head greatly depends on the storage. For systems with cascaded reservoirs, memory and CPU time requirements expand exponentially with problem size making DP unsuitable for hydro chains.

We report our experience with the proposed nonlinear model on a hydro chain based on a realistic hydro system with three cascaded reservoirs, considering a time horizon of $168 \mathrm{~h}$. As a new contribution to earlier studies, we address the short-term behaviour of head-dependent reservoirs in the hydro chain, studding it according to their position in the cascade and according to the new parameterisation defining the data for the hydro system. Previous studies presented reservoir optimal operation policies mainly for medium and long-term planning procedures, which typically encircle a time horizon of years on a weekly or monthly basis $[7,13,26,27]$. Moreover, the head change effect is taken into account in our study, which represents one of the main difficulties associated with the STHS problem, usually ignored when using linear models or neglected for hydro plants with a large storage capacity.

This paper is structured as follows. Section 2 provides the notation used throughout the paper along with the mathematical formulation of the STHS problem. Section 3 presents the NLP method for solving the STHS problem. Section 4 presents the numerical simulation results for the NLP method applied on a realistic cascaded hydro system. Section 5 outlines the conclusions.

\section{Problem formulation}

The notation used throughout the paper is described as follows:

$a_{i k} \quad$ natural inflow to reservoir $i$ during the period $k$

$\boldsymbol{A} \quad$ node-arc incident matrix

b right-hand side vector

$f \quad$ vector of coefficients for the linear term

$h_{i k} \quad$ head of plant $i$ during the period $k$

$h_{i}^{\max } \quad$ maximum head of plant $i$

$h_{i}^{\text {min }} \quad$ minimum head of plant $i$

$\boldsymbol{H} \quad$ Hessian matrix

$i \quad$ index of reservoirs

$k \quad$ index of periods in the time horizon

$K$ total number of periods in the time horizon

I total number of reservoirs

$l_{i k} \quad$ water level in reservoir $i$ during the period $k$

$l_{i}^{\max } \quad$ maximum water level in reservoir $i$

$l_{i}^{\text {min }} \quad$ minimum water level in reservoir $i$

$p_{i k} \quad$ power generation of plant $i$ during the period $k$

$q_{i k} \quad$ water discharge of plant $i$ during the period $k$

$q_{i}^{\max } \quad$ maximum discharge of plant $i$

$q_{i}^{\min } \quad$ minimum discharge of plant $i$

$\mathrm{s}_{i k} \quad$ water spillage by reservoir $i$ during the period $k$

$v_{i k} \quad$ water storage of reservoir $i$ at end of period $k$

$v_{i}^{\max } \quad$ maximum storage of reservoir $i$

$v_{i}^{\min } \quad$ minimum storage of reservoir $i$

$\boldsymbol{x} \quad$ vector of the flux variables corresponding to the arcs of the network

$\boldsymbol{x}^{\max } \quad$ upper bound vector 
$\boldsymbol{x}^{\mathrm{min}} \quad$ lower bound vector

$\eta_{i k} \quad$ efficiency of plant $i$ during the period $k$

$\eta_{i}^{\max } \quad$ maximum efficiency of plant $i$

$\eta_{i}^{\min } \quad$ minimum efficiency of plant $i$

$\pi_{k} \quad$ forecasted energy price during the period $k$

$\Psi_{i} \quad$ future value of water stored in reservoir $i$

The STHS problem is formulated as a nonlinear network constrained optimisation problem. The STHS problem can be stated as to find out the water discharges, $q_{i k}$, the water storages, $v_{i k}$, and the water spillages, $s_{i k}$, for each reservoir, $i=1$, $\ldots, I$, at all scheduling time periods, $k=1, \ldots, K$, over the time horizon considered, that optimise a performance criterion subject to the operational and physical constraints. Normally, the water storages at the end of the time horizon, $v_{i k}$, are valued for future operation use. The number of the variables for the problem, defining the size of the problem, can be determined as three times the number of reservoirs multiplied by the number of scheduling time periods [1]. For the case study used in this paper, the problem size is $3 \times 3 \times 168$, therefore existing 1512 variables.

In the STHS problem under consideration the performance criterion, the objective function, is a measure of the benefit obtained by the conversion of potential energy of the water available in the reservoirs into electric energy. The maximum of this objective function, satisfying all physical and operational constraints, is a blend of the benefit of the hydroelectric power generation due to discharges throughout the time horizon and the future benefit of the water left at the last period. Eq. (1) shows our objective function blending these two benefits. The objective function is composed of two terms. The first term expresses the economic value of the future use of the water stored in the reservoirs at the last period, $\Psi_{i}$. This term is considered if no final water storage requirement is specified as a constraint. The last term represents the profit with the hydro chain during the short-term time horizon, where $\pi_{k}$ is the forecasted energy price during the period $k$ and $p_{i k}$ is the power generation of plant $i$ during the period $k$.

In mathematical programming, the STHS problem can be formulated as to maximize

$J=\sum_{i=1}^{I} \Psi_{i}\left(v_{i k}\right)+\sum_{i=1}^{I} \sum_{k=1}^{K} \pi_{k} p_{i k}$

Subject to

- Water conservation equation for each reservoir:

$$
\begin{aligned}
& v_{i k}=v_{i, k-1}+a_{i k}-q_{i k}-s_{i k}+q_{i-1, k}+s_{i-1, k}, \\
& i=1, \ldots, I, \quad k=1, \ldots, K
\end{aligned}
$$

- Power generation equation:

$$
p_{i k}=P_{i k}\left(q_{i k}, \eta_{i k}\right), \quad i=1, \ldots, I, \quad k=1, \ldots, K
$$

- Head equation:

$$
h_{i k}=H_{i k}\left(l_{i k}, l_{i+1, k}\right), \quad i=1, \ldots, I, \quad k=1, \ldots, K
$$

- Water storage constraints:

$$
v_{i}^{\min } \leq v_{i k} \leq v_{i}^{\max }, \quad i=1, \ldots, I, \quad k=1, \ldots, K
$$

- Water discharge constraints:

$$
q_{i}^{\min } \leq q_{i k} \leq q_{i}^{\max }, \quad i=1, \ldots, I, \quad k=1, \ldots, K
$$

- Water spillage constraints:

$$
s_{i k} \geq 0, \quad i=1, \ldots, I, \quad k=1, \ldots, K
$$

The optimal value of the objective function given by Eq. (1) is determined subject to constraints of two kinds: equality constraints and inequality constraints or simple bounds on the variables. Eq. (2) corresponds to the water conservation equation for each reservoir and assumes that the water discharge from any upstream reservoir flows directly into the succeeding downstream reservoir with no time lag, where $v_{i k}$ is the water storage of reservoir $i$ at end of period $k, a_{i k}$ the natural inflow to reservoir $i$ during the period $k, q_{i k}$ the water discharge of plant $i$ during the period $k$, and $s_{i k}$ is the water spillage by reservoir $i$ during the period $k$. Although the water travel times or the water timedelays between cascaded reservoirs have not been considered, these can be easily taken into account if necessary. In Eq. (3) power generation, $p_{i k}$, is considered a function of water discharge and efficiency, $\eta_{i k}$, depending on the head, $h_{i k}$. In Eq. (4), the head is considered a function of the water levels in the upstream reservoir, $l_{i k}$, and of the downstream reservoir, $l_{i+1, k}$, depending on the water storages in the respectively reservoirs. In Eqs. (5) and (6), water storage and water discharge have lower and upper bounds. Here for each reservoir $i, v_{i}^{\max }$ is the maximum storage, $v_{i}^{\min }$ the minimum storage, $q_{i}^{\max }$ the maximum discharge, and $q_{i}^{\min }$ is the minimum discharge. In Eq. (7) a null lower bound is considered for water spillage. Normally, water spillage by the reservoirs occurs when without it the water storage exceeds its upper bound, so spilling is necessary to avoid damage. The initial water storages, $v_{i 0}$, and the inflows to reservoirs are assumed known.

\section{NLP method for the STHS problem}

NLP, namely quadratic programming, can be stated as to maximize

$\boldsymbol{J}(\boldsymbol{x})=(1 / 2) \boldsymbol{x}^{\mathrm{T}} \boldsymbol{H} \boldsymbol{x}+\boldsymbol{f}^{\mathrm{T}} \boldsymbol{x}$

Subject to

$\boldsymbol{A x}=\boldsymbol{b}$

$\boldsymbol{x}^{\min } \leq \boldsymbol{x} \leq \boldsymbol{x}^{\max }$

where for the STHS problem, viewed as a quadratic programming problem, $\boldsymbol{x}$ is the vector of the flux variables corresponding to the arcs of the underlying network structure in hydro chains. This vector consists of the water storages, $v_{i k}$, the water discharges, $q_{i k}$, and the water spillages, $s_{i k}$, for each reservoir, $i=1$, $\ldots, I$, and for each scheduling time period, $k=1, \ldots, K$. In Eq. (8), the function $\boldsymbol{J}(\cdot)$ is a quadratic function of variables, where $\boldsymbol{H}$ is the Hessian matrix and $\boldsymbol{f}$ is the vector of coefficients for the 
linear term. Eq. (9) corresponds to the equality constraints for the water conservation in Eq. (2), where $\boldsymbol{A}$ is the node-arc incident matrix and $\boldsymbol{b}$ is the right-hand side vector. Eq. (10) corresponds to the inequality constraints in Eqs. (5)-(7), where $\boldsymbol{x}^{\max }$ is the upper bound vector and $\boldsymbol{x}^{\mathrm{min}}$ is the lower bound vector.

In Eq. (3), the efficiency depends on the head. Assuming a linearization of this efficiency in plants, we have

$\eta_{i k}=\alpha_{i} h_{i k}+\eta_{i 0}$

where the parameters $\alpha_{i}$ and $\eta_{i 0}$ are given by

$\alpha_{i}=\left(\eta_{i}^{\max }-\eta_{i}^{\min }\right) /\left(h_{i}^{\max }-h_{i}^{\min }\right)$

$\eta_{i 0}=\eta_{i}^{\max }-\alpha_{i} h_{i}^{\max }$

In Eq. (12) parameter $\alpha_{i}$ of each plant $i$ depends on the extreme values for efficiency and head, where $\eta_{i}^{\max }$ is the maximum efficiency, $\eta_{i}^{\min }$ is the minimum efficiency, $h_{i}^{\max }$ is the maximum head and $h_{i}^{\text {min }}$ is the minimum head.

In Eq. (4), the water level depends on the water storage. Assuming linearization of the water level in reservoirs, we have

$l_{i k}=\beta_{i} v_{i k}+l_{i 0}$

where the parameters $\beta_{i}$ and $l_{i 0}$ are given by

$\beta_{i}=\left(l_{i}^{\max }-l_{i}^{\min }\right) /\left(v_{i}^{\max }-v_{i}^{\min }\right)$

$l_{i 0}=l_{i}^{\max }-\beta_{i} v_{i}^{\max }$

In Eq. (15) parameter $\beta_{i}$ of each reservoir $i$ depends on the extreme values for water level and storage, where $l_{i}^{\max }$ is the maximum water level, $l_{i}^{\min }$ is the minimum water level, $v_{i}^{\max }$ is the maximum water storage and $v_{i}^{\min }$ is the minimum water storage.

Substituting Eq. (11) into Eq. (3), we have

$p_{i k}=q_{i k}\left(\alpha_{i} h_{i k}+\eta_{i 0}\right)$

By substituting Eqs. (4) and (14) into Eq. (17), we have

$p_{i k}=q_{i k}\left(\alpha_{i} \beta_{i} v_{i k}+\alpha_{i} l_{i 0}-\alpha_{i} \beta_{i+1} v_{i+1, k}-\alpha_{i} l_{i+1,0}+\eta_{i 0}\right)$

Therefore, power generation becomes a nonlinear function of water discharge and water storage, given by

$p_{i k}=\alpha_{i} \beta_{i} q_{i k} v_{i k}-\alpha_{i} \beta_{i+1} q_{i k} v_{i+1, k}+\delta_{i} q_{i k}$

where the parameter $\delta_{i}$ is given by

$\delta_{i}=\alpha_{i}\left(l_{i 0}-l_{i+1,0}\right)+\eta_{i 0}$

The new parameter given as the product of $\alpha_{i}$ by $\beta_{i}$ is the most important coefficient for the power generation nonlinear relationship in our STHS problem, considering head dependence. This new parameter will have a crucial importance on the short-term behaviour of head-dependent reservoirs in a hydro chain, affecting optimal reservoirs storage trajectories according to their position in the cascade and according to the physical data defining the hydro system.

Eq. (19) is used to write the objective function into the form of Eq. (8). The parameter given as the product of $\alpha_{i}$ by $\beta_{i}$ multiplied by the forecasted energy price $\pi_{k}$ appears in the Hessian matrix, and the parameter $\delta_{i}$ multiplied by $\pi_{k}$ appears in the vector of coefficients for the linear term.

The Hessian matrix $\boldsymbol{H}$ is a symmetric matrix, hence all its eigenvalues are real numbers. The sum of the eigenvalues for our Hessian matrix is null because the trace, the sum of the main diagonal elements, is null as seen by Eq. (18), i.e., the Hessian matrix has positive and negative or null eigenvalues, meaning that the Hessian matrix is an indefinite matrix. Quadratic programming for a problem formulated as in Eqs. (8)-(10) can be classified due to the nature of the $\boldsymbol{H}$ matrix into the wellknown concave and the less well-known nonconcave quadratic programming [28], where the indefinite quadratic programming is the toughest and still is a research topic among specialists in global optimisation, being less general and taking advantage of the special mathematical structure exhibited in applications.

The objective function of our model is neither a concave nor a convex function, our new model for the STHS problem is an indefinite quadratic programming problem. Therefore, the optimal solution is not necessarily attained at a vertex of the feasible region, the polytope defined by the network constraints in Eq. (9) and box constraints in Eq. (10).

The following Karush-Kuhn-Tucker and the second order necessary conditions for $\boldsymbol{x}^{*}$ to be optimal apply to our STHS problem:

$\boldsymbol{A x} \boldsymbol{x}^{*}=\boldsymbol{b}$

$\boldsymbol{x}^{\min } \leq \boldsymbol{x}^{*} \leq \boldsymbol{x}^{\max }$

$\boldsymbol{\mu}^{\mathrm{T}}\left(\boldsymbol{x}^{\max }-\boldsymbol{x}^{*}\right)=0, \quad \boldsymbol{\mu} \geq 0$

$\boldsymbol{v}^{\mathrm{T}}\left(\boldsymbol{x}^{*}-\boldsymbol{x}^{\min }\right)=0, \quad \boldsymbol{v} \geq 0$

$\boldsymbol{H} \boldsymbol{x}^{*}+\boldsymbol{f}+\boldsymbol{A}^{\mathrm{T}} \boldsymbol{\lambda}-\boldsymbol{\mu}+\boldsymbol{v}=0$

$\boldsymbol{y}^{\mathrm{T}} \boldsymbol{H} \boldsymbol{y} \leq 0, \quad \boldsymbol{y} \in T\left(\boldsymbol{x}^{*}\right)$

where $T\left(x^{*}\right)$ is the tangent space at $\boldsymbol{x}^{*}$, given for our problem by $T\left(\boldsymbol{x}^{*}\right)=\left\{\boldsymbol{y}: \forall i \in R^{\mathrm{a}}\left(\boldsymbol{x}^{*}\right), \quad \boldsymbol{y}_{i}=0, \quad \boldsymbol{A} \boldsymbol{y}=0\right\}$

$R^{\mathrm{a}}\left(\boldsymbol{x}^{*}\right)$ is the set of active constraints at $\boldsymbol{x}^{*}$. Eqs. (21) and (22) are the conditions that ensure feasibility of the solution, Eqs. (23) and (24) are the complementary slackness conditions, Eq. (25) is the stationary condition and Eq. (26) is the second order necessary condition, meaning that the Hessian matrix is negative semidefinite with respect to the tangent space.

Exploiting the structure of our model for the STHS problem, we were able to conclude some proprieties. For instances, consider by hypothesis that $\boldsymbol{x}^{*}$ is a local maximizer but not a global maximizer for a problem formulated with Eqs. (8) and (9), which is the STHS problem without the box constraints. Hence, there exist a feasible $\boldsymbol{x}$ with a better objective function value for the problem, that is

$\boldsymbol{x}^{\mathrm{T}} \boldsymbol{H} \boldsymbol{x}-\boldsymbol{x}^{* \mathrm{~T}} \boldsymbol{H} \boldsymbol{x}^{*}+2 \boldsymbol{f}^{\mathrm{T}}\left(\boldsymbol{x}-\boldsymbol{x}^{*}\right)>\mathbf{0}$

noting that Eqs. (22)-(24) do not apply and by Eqs. (21) and (27) that $\boldsymbol{x}-\boldsymbol{x}^{*} \in T\left(\boldsymbol{x}^{*}\right)$ for any feasible $\boldsymbol{x}$, solving Eq. (25) in order to $f$ and substituting into Eq. (28), we have

$\left(\boldsymbol{x}-\boldsymbol{x}^{*}\right)^{\mathrm{T}} \boldsymbol{H}\left(\boldsymbol{x}-\boldsymbol{x}^{*}\right)>\mathbf{0}$ 
due to the second order necessary optimality condition, results a contradiction of the hypothesis. Hence, $\boldsymbol{x}^{*}$ is a local maximizer if and only if it is a global maximizer for the STHS problem without the box constraints, this is known as uniextremality.

Now, consider by hypothesis that $\boldsymbol{x}^{*}$ is a global maximizer for the STHS problem with the box constraints, the difference of objective function values between a feasible point $x$ and $x^{*}$ is given by

$\boldsymbol{J}(\boldsymbol{x})-\boldsymbol{J}\left(\boldsymbol{x}^{*}\right)=\left(\boldsymbol{x}-\boldsymbol{x}^{*}\right)^{\mathrm{T}} \boldsymbol{H}\left(\boldsymbol{x}-\boldsymbol{x}^{*}\right)+2(\boldsymbol{\mu}-\boldsymbol{v})^{\mathrm{T}}\left(\boldsymbol{x}-\boldsymbol{x}^{*}\right)$

considering Eqs. (23) and (24), we have

$2(\boldsymbol{v}-\boldsymbol{\mu})^{\mathrm{T}}\left(\boldsymbol{x}-\boldsymbol{x}^{*}\right) \geq \mathbf{0}$

and considering Eq. (30), we have

$\left(\boldsymbol{x}-\boldsymbol{x}^{*}\right)^{\mathrm{T}} \boldsymbol{H}\left(\boldsymbol{x}-\boldsymbol{x}^{*}\right) \leq 2(\boldsymbol{v}-\boldsymbol{\mu})^{\mathrm{T}}\left(\boldsymbol{x}-\boldsymbol{x}^{*}\right)$

The Hessian matrix is an indefinite matrix, Eq. (32) implies $R^{\mathrm{a}}\left(\boldsymbol{x}^{*}\right) \neq \phi$ to ensure $(\boldsymbol{v}-\boldsymbol{\mu})^{\mathrm{T}}\left(\boldsymbol{x}-\boldsymbol{x}^{*}\right)>\mathbf{0}$. Due to this the global maximizer is attained at the boundary of the feasible region, not necessarily at a vertex as it happens if the problem is transformed into an LP problem relaxation or when $\boldsymbol{H}$ is positive definite, but it is a good initial guess to start by such a vertex and consider the neighbourhood around the vertex a good basin for achieving a superior objective function value.

We consider a starting point given by an LP problem and using quadratic programming we check for a superior objective function value. In our case studies we always arrive at convergence to a superior solution. The robustness features of optimisation methods are important and far from trivial. Nonetheless, some authors consider that robustness should be in part sacrificed to achieve fastness [29].

\section{Practical example}

The proposed NLP method, considering the head change effect, has been applied on a cascaded hydro system based on a real case. The hydro system is shown in Fig. 1.

This example consists of three cascaded reservoirs, considering natural inflow only on the first reservoir as shown in Fig. 2.

The numerical simulation was performed on a $1.6 \mathrm{GHz}$-based processor with $512 \mathrm{MB}$ of RAM. The scheduling time horizon is $168 \mathrm{~h}$. The forecasted energy price considered over the time horizon is shown in Fig. 3 (\$ is a symbolic economic quantity).

The deregulation of the electricity markets brings uncertainty to energy prices. A good forecasting tool provides a risk hedging mechanism for generating companies against price volatility. In addition, a generating company can develop an appropriate bidding strategy to maximize its own profit with an accurate price forecast, which represents an advantage facing competition. Several forecasting procedures are available for predicting energy prices, such as time series [30], neural networks [31], or neural networks combined with fuzzy logic [32], but for the STHS problem the prices are considered as deterministic input data.

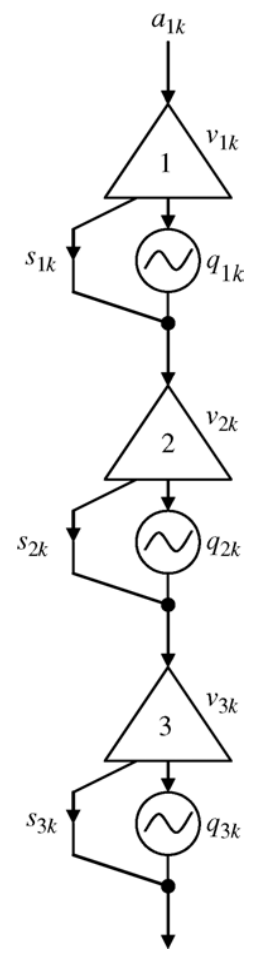

Fig. 1. Hydro system with three cascaded reservoirs.

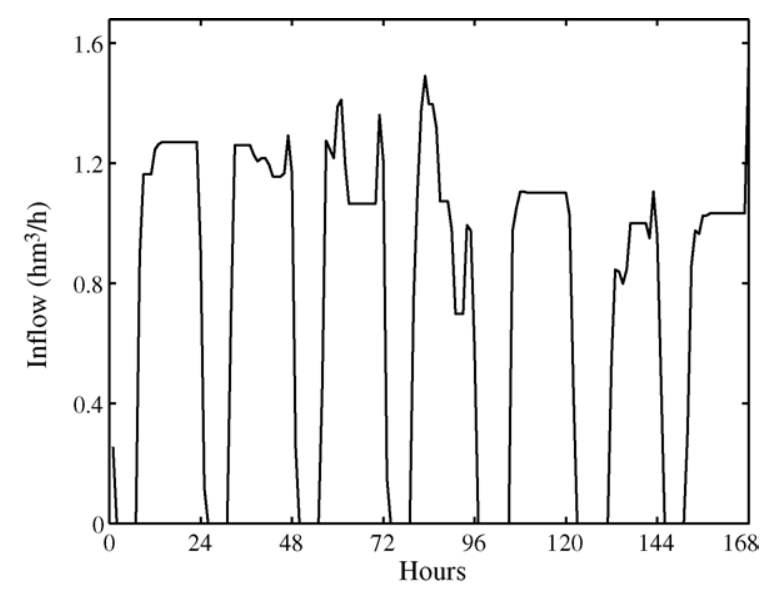

Fig. 2. Inflow on the first reservoir.

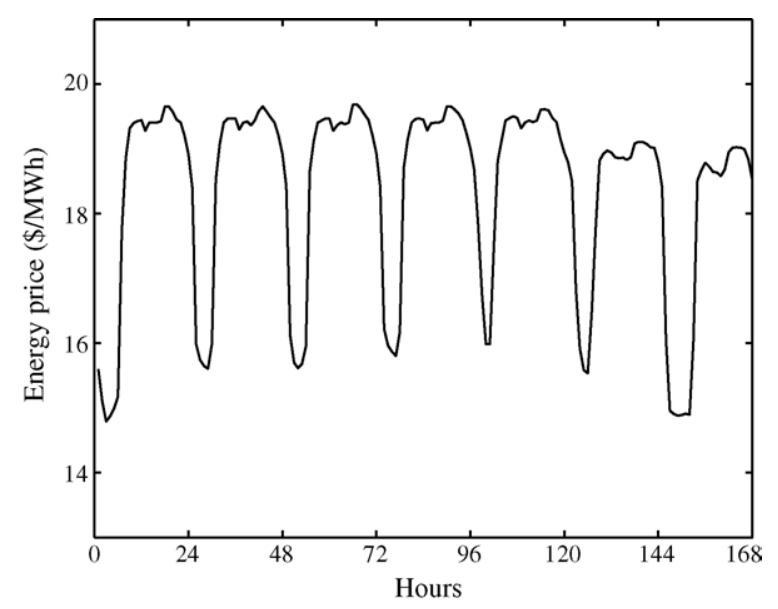

Fig. 3. Forecasted energy price. 


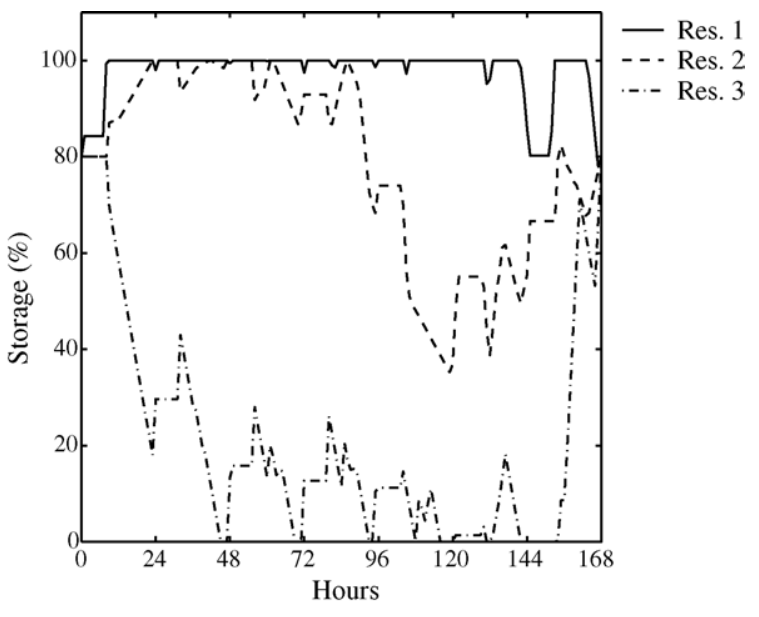

Fig. 4. Optimal reservoir storage trajectories for case 1.

Final water storage in reservoirs is constrained so the water storage in the reservoirs at the last period is fixed. Consequently, the future values of water stored in reservoirs are not considered in this example. The final water storage in reservoirs is considered equal to the value at the beginning of the scheduling horizon, chosen to be $80 \%$ of the maximum storage.

This example is divided into four cases to evaluate the parameterisation effect on the short-term behaviour of the headdependent hydro chain. The cases are defined by:

- Case 1: $\alpha_{1} \beta_{1}>\alpha_{2} \beta_{2}>\alpha_{3} \beta_{3}$;

- Case 2: $\alpha_{2} \beta_{2}>\alpha_{1} \beta_{1}>\alpha_{3} \beta_{3}$;

- Case 3: $\alpha_{1} \beta_{1}>\alpha_{3} \beta_{3}>\alpha_{2} \beta_{2}$;

- Case 4: $\alpha_{3} \beta_{3}>\alpha_{2} \beta_{2}>\alpha_{1} \beta_{1}$.

\subsection{Case 1}

The optimal reservoir storage and head trajectories for this case are shown respectively in Figs. 4 and 5.

The optimisation postpones power generation in the initial periods on all plants to quickly achieve appropriated reservoir storage levels, pulling up the storage trajectories of the two first

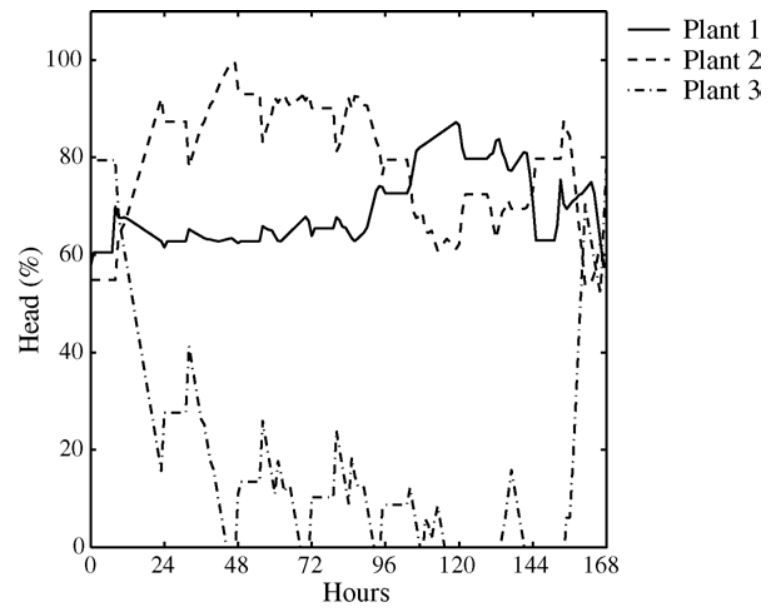

Fig. 5. Optimal plant head trajectories for case 1.

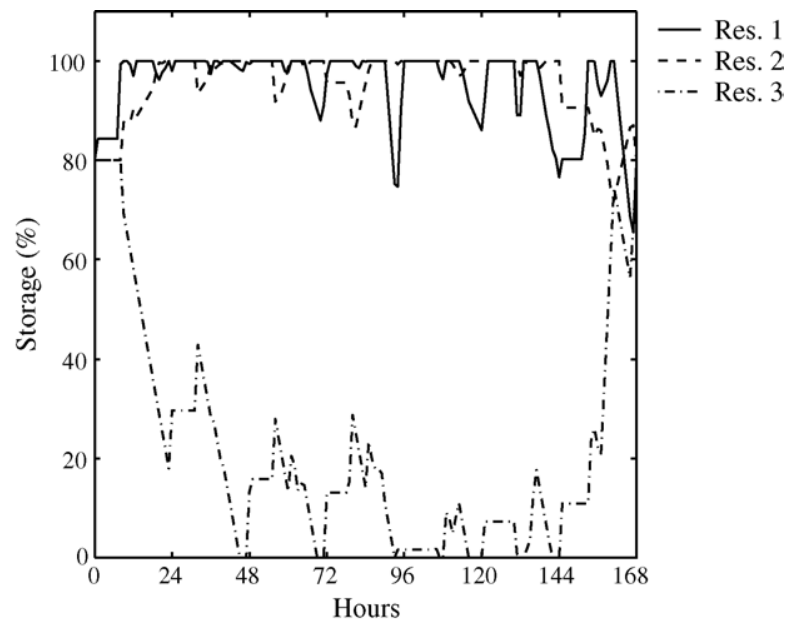

Fig. 6. Optimal reservoir storage trajectories for case 2.

reservoirs, in order to benefit the generation's efficiency of the two first plants, opposing to the change in the storage trajectory of the third reservoir. Consequently, implies that the reservoirs play a completely different role in the system depending on their relative position in the hydro chain. Also, compared with an LP method that ignores head dependence, an increase in profit of $3.21 \%$ is achieved.

\subsection{Case 2}

The optimal reservoir storage and head trajectories for this case are shown respectively in Figs. 6 and 7.

As in the first case the optimisation pulls up the storage trajectories of the first and the second reservoirs in order to benefit the generation's efficiency of the first and the second plants, opposing to the change in the storage trajectory of the third reservoir. Hence, it is the generation's efficiency in the third reservoir that is once again sacrificed, comparing with case 1 , but due to the inequality $\alpha_{2} \beta_{2}>\alpha_{1} \beta_{1}>\alpha_{3} \beta_{3}$ the second reservoir is nearer to the maximum water storage than the first reservoir. In this case, an increase in profit of $3.58 \%$ relative to the LP method is achieved.

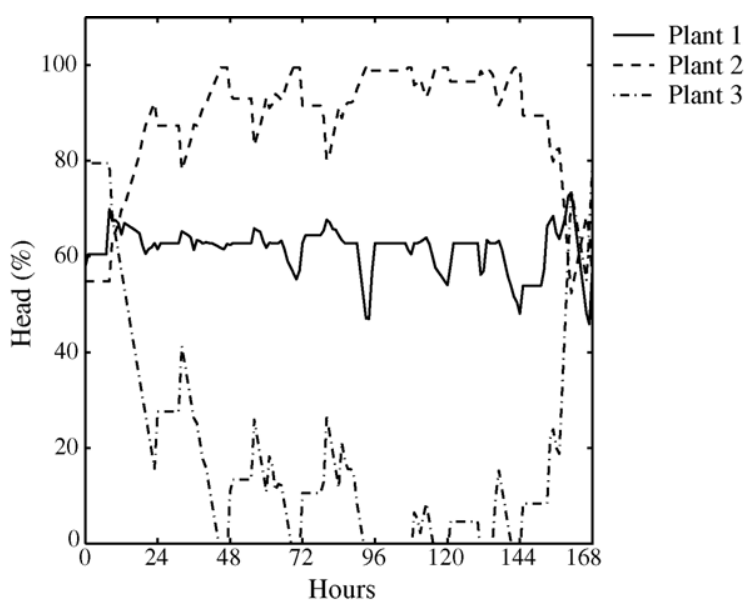

Fig. 7. Optimal plant head trajectories for case 2. 


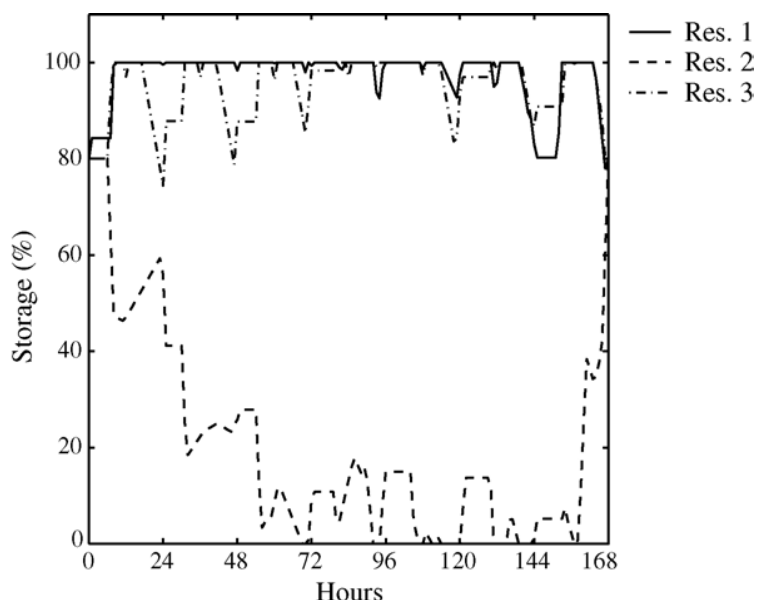

Fig. 8. Optimal reservoir storage trajectories for case 3.

\subsection{Case 3}

The optimal reservoir storage and head trajectories for this case are shown respectively in Figs. 8 and 9.

In case 3 , due to the inequalities $\alpha_{1} \beta_{1}>\alpha_{3} \beta_{3}>\alpha_{2} \beta_{2}$, the optimisation pulls up the storage trajectories of the first and the third reservoirs in order to benefit the generation's efficiency of the first and the third plants, opposing to the change in the storage trajectory of the second reservoir. Hence, it is the generation's efficiency in the second reservoir that is sacrificed. In this case, an increase in profit of $5.42 \%$ relative to the LP method is achieved.

\subsection{Case 4}

The optimal reservoir storage and head trajectories for this case are shown respectively in Figs. 10 and 11.

In case 4 , due to the inequalities $\alpha_{3} \beta_{3}>\alpha_{2} \beta_{2}>\alpha_{1} \beta_{1}$, the optimisation pulls up the storage trajectories in all reservoirs in order to benefit the generation's efficiency of their plants where the third reservoir is the nearest to the maximum water storage. The first reservoir has the lowest coefficient for the power generation nonlinear relationship of all reservoirs. Hence, it does not

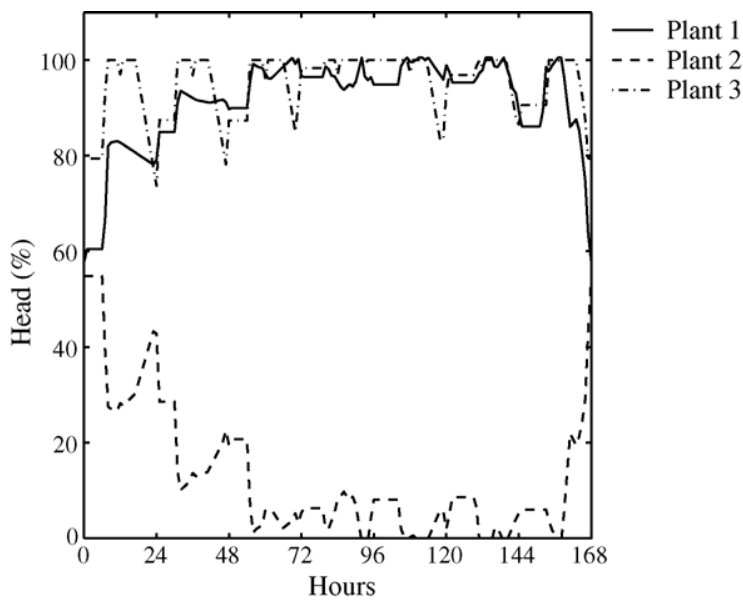

Fig. 9. Optimal plant head trajectories for case 3.

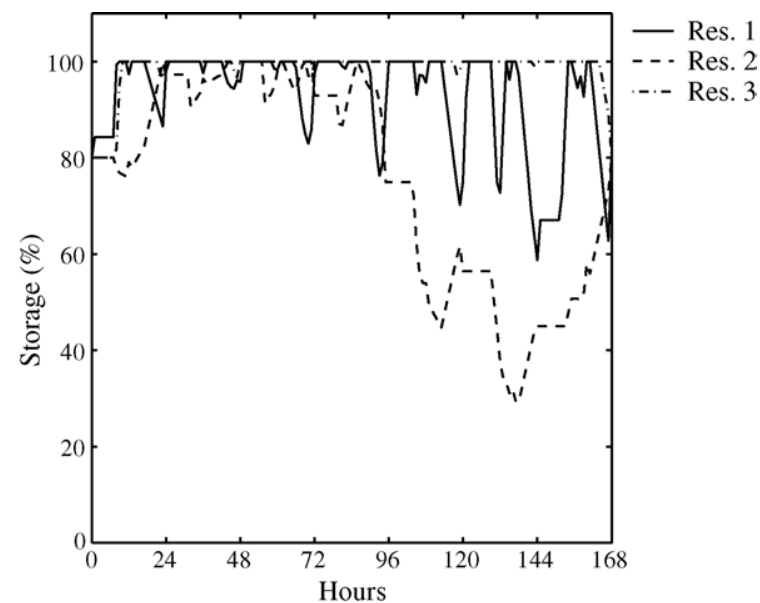

Fig. 10. Optimal reservoir storage trajectories for case 4 .

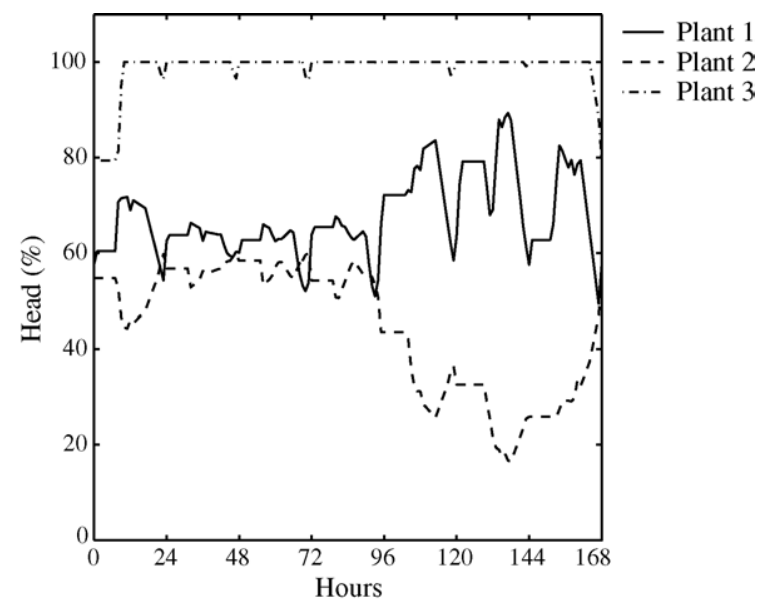

Fig. 11. Optimal plant head trajectories for case 4 .

assume the behaviours observed in previous cases. In this case the profit achieved assumes its highest value with an increase of $7.86 \%$ relative to the LP method.

Table 1 summarizes an overall comparison between the results obtained by the classical LP method that ignores head dependence, and the proposed NLP method.

On the one hand, the computational effort is negligible with the proposed NLP method, extra CPU time is about $0.14 \mathrm{~s}$. Hence, it is an efficient method for the STHS problem achieving fast convergence for all cases tested, therefore providing better results for head-dependent hydro chains.

Table 1

Comparison of LP with the proposed NLP method

\begin{tabular}{llll}
\hline & Profit $(\$)$ & \% Increase & CPU time (s) \\
\hline LP & 1055999 & - & 0.21 \\
NLP (case 1) & 1089881 & 3.21 & 0.35 \\
NLP (case 2) & 1093851 & 3.58 & 0.35 \\
NLP (case 3) & 1113283 & 5.42 & 0.35 \\
NLP (case 4) & 1139018 & 7.86 & 0.35 \\
\hline
\end{tabular}


On the other hand, different parameter settings were considered in order to highlight the behaviour features of the reservoirs and the influence of the new hydro chain parameters in STHS. As a consequence, it is possible to conclude that the physical data defining the new hydro system parameters used in the nonlinear model have a significant effect on the behaviour of head-dependent reservoirs in a hydro chain, implying different optimal storage trajectories to the reservoirs accordingly, not only with their relative position in the cascade, but also with the parameterisation defining the data for the hydro system.

\section{Conclusion}

This paper deals with the STHS problem viewed as an indefinite quadratic programming problem with continuous variables. The proposed NLP method applied to the STHS problem is successful, providing a higher profit in comparison with classic optimisation methods based on LP that ignore head dependence and nonlinearity in the objective function, with negligible extra computational effort required. A better short-term management of the conversion of the potential energy available in the reservoirs into electric energy, considering head dependency, is crucial for hydroelectric utilities to face competitiveness in nowadays profit-based environment. An example based on a real cascaded hydro system is used to prove as a new contribution to earlier studies that the physical data defining the hydro chain parameters used in the nonlinear model have a significant effect on the behaviour of reservoirs in a hydro chain, implying different optimal storage trajectories to the reservoirs accordingly, not only with their relative position in the cascade, but also with the new parameterisation defining the data for the hydro system. Hence, we conclude as the main contribution of this paper for the short-term management of head-dependent hydro chains that there is no clear and definitive rule about what is the volume of the water to store in each reservoir at each period to achieve the optimum STHS based only on the reservoirs relative position.

\section{References}

[1] A.H. Mantawy, S.A. Soliman, M.E. El-Hawary, The long-term hydroscheduling problem-a new algorithm, Electr. Power Syst. Res. 64 (1) (2003) 67-72.

[2] V.M.F. Mendes, S.J.P.S. Mariano, J.P.S. Catalão, L.A.F.M. Ferreira, Emission constraints on short-term schedule of thermal units, in: Proceedings of the 39th International Universities Power Engineering Conference, Bristol, UK, 2004, pp. 1068-1072.

[3] L.A.F.M. Ferreira, T. Andersson, C.F. Imparato, T.E. Miller, C.K. Pang, A. Svoboda, A.F. Vojdani, Short-term resource scheduling in multi-area hydro-thermal power systems, Electr. Power Energy Syst. 11 (3) (1989) 200-212.

[4] J.P.S. Catalão, S.J.P.S. Mariano, V.M.F. Mendes, L.A.F.M. Ferreira, Scheduling of head-sensitive cascaded hydro systems: a comparison based on numerical simulation results, in: Proceedings of the Fourth IASTED International Conference on Power and Energy Systems, Rhodes, Greece, 2004, pp. 418-423.

[5] A.I. Cohen, V.R. Sherkat, Optimization-based methods for operations scheduling, Proc. IEEE 75 (12) (1987) 1574-1591.

[6] S.M. Amado, C.C. Ribeiro, Short-term generation scheduling of hydraulic multi-reservoir multi-area interconnected systems, IEEE Trans. Power Syst. 2 (3) (1987) 758-763.
[7] C. Lyra, L.R.M. Ferreira, A multiobjective approach to the short-term scheduling of a hydroelectric power system, IEEE Trans. Power Syst. 10 (4) (1995) 1750-1755.

[8] A. Arce, T. Ohishi, S. Soares, Optimal dispatch of generating units of the Itaipú hydroelectric plant, IEEE Trans. Power Syst. 17 (1) (2002) $154-158$.

[9] J.M. Pursimo, H.K. Antila, M.K. Vilkko, P.A. Lautala, A short-term scheduling for a hydropower plant chain, Electr. Power Energy Syst. 20 (8) (1998) 525-532.

[10] R. Naresh, J. Sharma, Short term hydro scheduling using two-phase neural network, Electr. Power Energy Syst. 24 (7) (2002) 583-590.

[11] M. Basu, Hopfield neural networks for optimal scheduling of fixed head hydrothermal power systems, Electr. Power Syst. Res. 64 (1) (2003) $11-15$.

[12] P. Chen, H. Chang, Genetic aided scheduling of hydraulically coupled plants in hydro-thermal coordination, IEEE Trans. Power Syst. 11 (2) (1996) 975-981.

[13] P.T. Leite, A.A.F.M. Carneiro, A.C.P.L.F. Carvalho, Energetic operation planning using genetic algorithms, IEEE Trans. Power Syst. 17 (1) (2002) 173-179.

[14] D. Sjelvgren, S. Andersson, T. Andersson, U. Nyberg, T.S. Dillon, Optimal operations planning in a large hydro-thermal power system, IEEE Trans. Power Apparatus Syst. PAS-102 (11) (1983) 3644-3651.

[15] P.E.C. Franco, M.F. Carvalho, S. Soares, A network flow model for short-term hydro-dominated hydrothermal scheduling problems, IEEE Trans. Power Syst. 9 (2) (1994) 1016-1022.

[16] A.R.L. Oliveira, S. Soares, L. Nepomuceno, Short term hydroelectric scheduling combining network flow and interior point approaches, Electr. Power Energy Syst. 27 (2) (2005) 91-99.

[17] E.B. Hreinsson, Optimal short term operation of a purely hydroelectric system, IEEE Trans. Power Syst. 3 (3) (1988) 1072-1077.

[18] M.R. Piekutowski, T. Litwinowicz, R.J. Frowd, Optimal short-term scheduling for a large-scale cascaded hydro system, IEEE Trans. Power Syst. 9 (2) (1994) 805-811.

[19] C. Li, E. Hsu, A.J. Svoboda, C. Tseng, R.B. Johnson, Hydro unit commitment in hydro-thermal optimisation, IEEE Trans. Power Syst. 12 (2) (1997) 764-769.

[20] M. Christoforidis, B. Awobamise, S. Tong, M. Aganagic, R.J. Frowd, F.A. Rahimi, Short-term hydro generation and interchange contract scheduling for Swiss rail, IEEE Trans. Power Syst. 11 (1) (1996) 274-280.

[21] O. Nilsson, D. Sjelvgren, Mixed-integer programming applied to shortterm planning of a hydro-thermal system, IEEE Trans. Power Syst. 11 (1) (1996) 281-286.

[22] X. Guan, A. Svoboda, C. Li, Scheduling hydro power systems with restricted operating zones and discharge ramping constraints, IEEE Trans. Power Syst. 14 (1) (1999) 126-131.

[23] G.W. Chang, M. Aganagic, J.G. Waight, J. Medina, T. Burton, S. Reeves, M. Christoforidis, Experiences with mixed integer linear programming based approaches on short-term hydro scheduling, IEEE Trans. Power Syst. 16 (4) (2001) 743-749.

[24] A.J. Conejo, J.M. Arroyo, J. Contreras, F.A. Villamor, Self-scheduling of a hydro producer in a pool-based electricity market, IEEE Trans. Power Syst. 17 (4) (2002) 1265-1272.

[25] E. Ni, X. Guan, R. Li, Scheduling hydrothermal power systems with cascaded and head-dependent reservoirs, IEEE Trans. Power Syst. 14 (3) (1999) 1127-1132.

[26] S. Soares, A.A.F.M. Carneiro, Optimal operation of reservoirs for electric generation, IEEE Trans. Power Delivery 6 (3) (1991) 1101-1107.

[27] G. Cruz Jr., S. Soares, Non-uniform composite representation of hydroelectric systems for long-term hydrothermal scheduling, IEEE Trans. Power Syst. 11 (2) (1996) 702-707.

[28] C.A. Floudas, V. Visweswaran, in: R. Horst, P.M. Pardalos (Eds.), Quadratic Optimization, Handbook of Global Optimization, Kluwer Academic Publishers, 1995, pp. 217-270.

[29] A. Neumaier, in: A. Iserles (Ed.), Complete Search in Continuous Global Optimization and Constraint Satisfaction, Acta Numerica, Cambridge University Press, 2004, pp. 271-369. 
[30] F.J. Nogales, J. Contreras, A.J. Conejo, R. Espínola, Forecasting nextday electricity prices by time series models, IEEE Trans. Power Syst. 17 (2) (2002) 342-348.

[31] H.Y. Yamin, S.M. Shahidehpour, Z. Li, Adaptative short-term electricity price forecasting using artificial neural networks in the restruc- tured power markets, Electr. Power Energy Syst. 26 (8) (2004) 571581.

[32] Y.-Y. Hong, C.-F. Lee, A neuro-fuzzy price forecasting approach in deregulated electricity markets, Electr. Power Syst. Res. 73 (2) (2005) $151-157$. 